\title{
Reduction of oxidative stress in adjuvant arthritis. Comparison of efficacy of two pyridoindoles: stobadine dipalmitate and

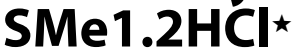

\author{
Silvester Ponist ${ }^{凶}$, Danica Mihalova ${ }^{1}$, Viera Jancinova1, Vladimír Snirc1, Olga Ondrejickova1, \\ Cinzia Mascia2, Giuseppe Poli², Maria Stancikova ${ }^{3}$, Radomir Nosal ${ }^{1}$ and Katarína Bauerová1 ${ }^{1}$
}

The aim of this study was to evaluate the therapeutic potential of oxidative stress (OS) reduction by using pyridoindole (PI) antioxidants in adjuvant arthritis (AA). The substances tested were stobadine dipalmitate (STB) and SMe1. AA was used as animal model. The experiments included healthy animals, control arthritic animals and arthritic animals with administration of $\mathrm{PI}$ in the oral daily dose of $15 \mathrm{mg} / \mathrm{kg}$ b.m during 28 experimental days. The rats were sacrificed on day 28. Clinical and biochemical parameters were determined. The effect of $\mathrm{PI}$ administration was evaluated on the basis of the following parameters: (a) arthritis (volume of hind paws - HPW, change of animal body mass - CBM), (b) OS (chemiluminescence of whole blood - CWB, levels of thiobarbituric acid reacting substance - TBARS and of HNE- and MDA-protein adducts in plasma and activity of Y-glutamyltransferase (GGT) in hind paw joint homogenates). The PI studied significantly increased the CBM of animals and corrected the HPW. STB also significantly decreased the activity of GGT in joint homogenates. SMe1 was more effective in decreasing plasmatic TBARS levels, but STB was more effective in reducing plasmatic HNE- and MDA-protein adducts. The assay for HNE- and MDA-adducts in plasma as a function of time was applied for the first time in AA. STB markedly decreased spontaneous and PMA-stimulated CWB and reduced neutrophil count. In summary, STB was more effective than SMe1 in reducing OS in AA. Our results showed that the reduction of OS in arthritis also corrected the clinical manifestations of the disease.

Keywords: oxidative stress, arthritis, inflammation, pyridoindoles, HNE- and MDA-protein adducts, whole blood chemiluminescence, neutrophil

Received: 28 February, 2010; revised: 13 May, 2010; accepted: 09 June, 2010; available on-line: 10 June, 2010

\section{INTRODUCTION}

Rheumatoid arthritis (RA) is frequent chronic autoimmune disease. In general, the disease progresses and often leads to disability. It can shorten the patient's life span by 10 years. The cause of the disease is multifactorial, including genetic predisposition. It is characterized by typical chronic inflammation and initiated and maintained by autoimmune mechanisms. The pathogenesis of $\mathrm{RA}$ is associated predominantly with the formation of free radicals and proinflammatory cytokines at the site of inflammation. The inflammatory process develops in the tissue of the synovium; primary sources of reactive oxidative species (ROS) in RA are leukocytes, which are recruited to accumulate within the synovium. ROS can be produced by activated macrophages in the synovial membrane and by activated neutrophils in the synovial cavity (Firestein, 2003; Bauerova et al., 2009). The immune mechanisms responsible for the development of rheumatoid arthritis are well known. To date, however, we have less information about the role of systemic oxidative stress in RA and the efficacy of therapies based on reducing oxidative stress. In our experiments with the adjuvant arthritis (AA) model (Bauerova et al., 2004; 2005a; 2005b; 2008a; 2008b; 2009; Gvozdjakova et al., 2004; Kogan et al., 2005; Nosal et al., 2007; Drabikova et al., 2009; Jancinova et al., 2009; Rovensky et al., 2009; Sotnikova et al., 2009; Strosova et al., 2008) we therefore focused on monitoring oxidative stress and potential therapies with substances possessing anti-oxidative and/ or anti-inflammatory properties. For our experiment we had chosen substances with anti-oxidative properties and low toxicity. Stobadine dipalmitate (STB) belongs to the group of naturally occurring carbolines (pyridoindoles, PI), tricyclic compounds of indole structure with a side ring comprising another nitrogen atom. Unlike $\alpha$ - and $\beta$-carbolines, it does not reveal any obvious toxic effects and possesses an antioxidant activity. STB, (-)-cis2,8-dimethyl-2,3,4,4a,5,9b-hexahydro- $1 H$-pyrido[4,3b]indole, was developed and synthesized in a search for new antidysrhythmic drugs at the Institute of Experimental Pharmacology Slovak Academy of Sciences in Bratislava, Slovakia in 1983 (Stolc et al., 1983; Juranek et al., 2010). Later also the pyridoindole (PI) derivative SMe1.2 $\mathrm{HCl}$ : 8-methoxy-2,3,4,4a, 5,9b-hexahydro- $1 H$-pyrido[4,3b]indole was synthesized at our institute. The first results with STB in rat adjuvant arthritis were obtained when its protective effect against indomethacin-induced gastroenteropathy was evaluated (Bauerova et al., 2004). The aim

S.P. e-mail: exfasipo@savba.sk; K.B. e-mail: exfakbau@savba.sk ${ }^{\star}$ A preliminary report on the same subject was presented as poster at the European Meeting of SFRR in Rome, Italy, 26-29 August, 2009 and partially published in Bauerova et al., 2006.

Abbreviations: AA, adjuvant arthritis; b.m., body mass; CBM, change of body mass; GGT, $\gamma$-glutamyltransferase; $\gamma$-GPN $\gamma$-glutamyl-4-nitroanilide; HPV, hind paw volume; HNE, 4-hydroxy2-nonenal; HRP, horseradish peroxidase; MDA, malondialdehyde; $\mathrm{PI}$ pyridoindoles; OS, oxidative stress; PMA, phorbolmyristate acetate; RA, rheumatoid arthritis; RNS, reactive nitrogen species; ROS, reactive oxygen species; STB, stobadine dipalmitate; SMe1, SMe1.2HCl; S.E.M., standard error of mean; TBA, thiobarbituric acid; TBARS, thiobarbituric acid reacting substance. 
of the present work was to evaluate the efficacy of two pyridoindoles, STB and SMe1, in the reduction of oxidative stress in adjuvant arthritis.

\section{MATERIALS AND METHODS}

Experimental design. After approval by the local ethics committee, AA was induced in male Lewis rats (Breeding Farm Dobra Voda, Slovakia), weighing 150-170 g each, by a single intradermal injection of heat-inactivated Mycobacterium butyricum (MB) in incomplete Freund's adjuvant (Difco Laboratories, Detroit, MI, USA). In each experimental group, 6-8 animals were used. The experiment included healthy animals as reference controls (CO), arthritic animals without any drug administration (AA), and arthritic animals with the administration of stobadine dipalmitate (AA-STB) or its derivative $\mathrm{SMe} 1.2 \mathrm{HCl}$ (AA-SMe1) in an oral daily dose of $15 \mathrm{mg} / \mathrm{kg}$ b.m. for 28 days. All parameters were monitored on day 28, except HNE- and MDA-protein adducts. The HNE- and MDA-protein adducts were measured on days: 7, 14, 21 and 28. The day with the highest level for HNE- and MDA-protein adducts in the plasma (day 14) was chosen for evaluation of PI treatment.

Clinical parameters. We monitored the clinical parameters CBM and HPV twice a week. CBM was calculated as the difference of the body mass measured on day 28 and the body mass measured at the beginning of the experiment. The HPV increase was calculated as the percentage increase of HPV on day 28 in comparison to the beginning of the experiment.

$\gamma$-Glutamyltransferase. The parameter of inflammation - GGT in the joint from the hind paw (cartilage and soft tissue without bone) - was determined at the end of the experiment, on day 28. The activity of GGT was measured by the method of Orlowski and Meister (1970), modified by Ondrejickova et al. (1993). Samples were homogenized in buffer 1:9 (w/v) (composition: $2 \mathrm{mM} \mathrm{NaH} \mathrm{HPO}_{4}, 20 \mathrm{mM} \mathrm{Na} \mathrm{PO}_{4}, 15 \mathrm{mM}$ EDTA, $68 \mathrm{mM} \mathrm{NaCl}, \mathrm{pH}$ 8.1) by Ultra Turax TP 18/10 Janke \& Kunkel (Germany) for $1 \mathrm{~min}$ at $0^{\circ} \mathrm{C}$. Substrates $(8.7$

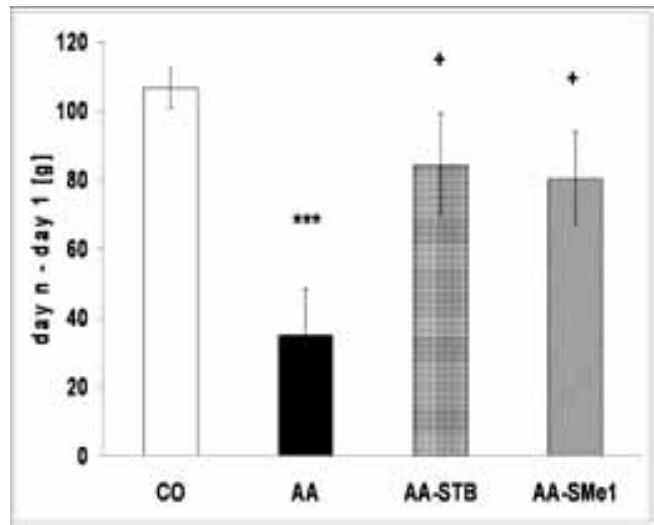

Figure 1. Effect of pyridoindoles on changes in body mass of arthritic rats.

The data were expressed as arithmetic mean with S.E.M. Each group contained 6-8 animals. Statistical significance was evaluated using unpaired Student's $t$-test: ${ }^{* * *} P<0.001$ with respect to control healthy animals; $+P<0.05$ with respect to untreated arthritic animals. The experiment included healthy intact animals as reference controls (CO), arthritic animals without any drug administration (AA), and arthritic animals with the administration of stobadine dipalmitate (AA-STB) or its derivative SMe1.2 HCI (AASMe1).

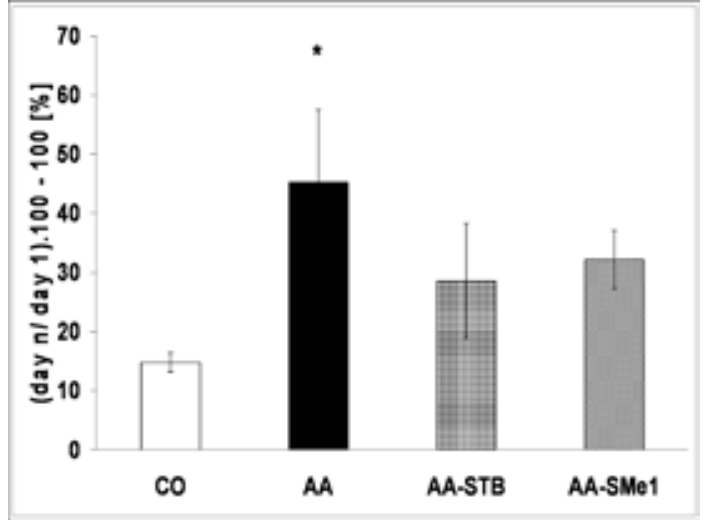

Figure 2. Effect of pyridoindoles on hind paw volume of arthritic rats.

The data were expressed as arithmetic mean with S.E.M. Each group contained 6-8 animals. Statistical significance was evaluated using unpaired Student's $t$-test: ${ }^{*} P<0.05$ with respect to control healthy animals. Groups of animals are the same as in Fig. 1.

$\mathrm{mM} \gamma$-glutamyl-4-nitroanilide ( $\gamma$-GPN); $44 \mathrm{mM}$ methionine) were dissolved in 65\% isopropyl alcohol with final concentrations in reaction mixture: $2.5 \mathrm{mM} \gamma$-GPN; 12.6 $\mathrm{mM}$ methionine. After incubation for $60 \mathrm{~min}$ at $37^{\circ} \mathrm{C}$ the reaction was stopped by adding $2.3 \mathrm{ml}$ of cold methanol and the tubes were centrifuged for $20 \mathrm{~min}$ at 5000 rpm. Absorbance of supernatant was measured by spectrophotometer Specord 40 (Jena, Germany) in $0.5 \mathrm{~cm}$ cuvette at $406 \mathrm{~nm}$. Reaction mixtures in the absence of either the substrate or acceptor were used as reference samples.

Thiobarbituric acid reactive substances. The reaction with TBA occurs by attack of the monoenolic form of MDA on the active methylene groups of TBA. Visible and ultraviolet spectrophotometry of the pigment confirms the primary maximum at $535 \mathrm{~nm}$. TBARS were measured in heparinized blood plasma (Brown \& Kelly, 1996). The amount of $750 \mu$ l of $0.67 \%$ thiobarbituric acid, $750 \mu \mathrm{l}$ of $20 \%$ trichloroacetic acid, $350 \mu$ l of phosphate buffer ( $\mathrm{pH} 7.4$ ) were added to $150 \mu \mathrm{l}$ of plasma, then mixed and incubated in a water bath at $90^{\circ} \mathrm{C}$ for $30 \mathrm{~min}$. The reaction was stopped by dipping the test tubes into ice for $10 \mathrm{~min}$. Samples were centrifuged at $3000 \mathrm{rpm}$. The supernatant was removed and absorbance measured at 535 $\mathrm{nm}$ in a $0.5 \mathrm{~cm}$ cuvette.

HNE- and MDA-protein adducts. The presence of oxidative damage was assessed in the plasma by determining the fluorescent adducts formed between peroxidation-derived aldehydes and plasma proteins. In particular, two types of protein-aldehyde adducts were determined: HNE-protein adducts (355 nm excitation, $460 \mathrm{~nm}$ emission) and MDA-protein adducts (390 nm excitation, $460 \mathrm{~nm}$ emission) (Biasi et al., 1995). Serum $(100 \mu \mathrm{l})$ was pipetted into a $10-\mathrm{ml}$ testtube with a glass stopper and $4 \mathrm{ml}$ of ethanol/ether $(3: 1, \mathrm{v} / \mathrm{v})$ was vigorously poured into it. The testtube was shaken with a vortex shaker and 5 min later centrifuged at $3000 \mathrm{rpm}$ for $5 \mathrm{~min}$. The solvents were discarded and further $4 \mathrm{ml}$ of the solvent mixture was added to the sediment, which was thoroughly rinsed. The sediment obtained as above was dissolved in $2 \mathrm{ml}$ of distilled water with a small amount of $0.5 \%$ sodium dodecyl sulphate (Tsuchida et al., 1985). The concentration of proteins in all test tubes was measured using Lowry`s method. 


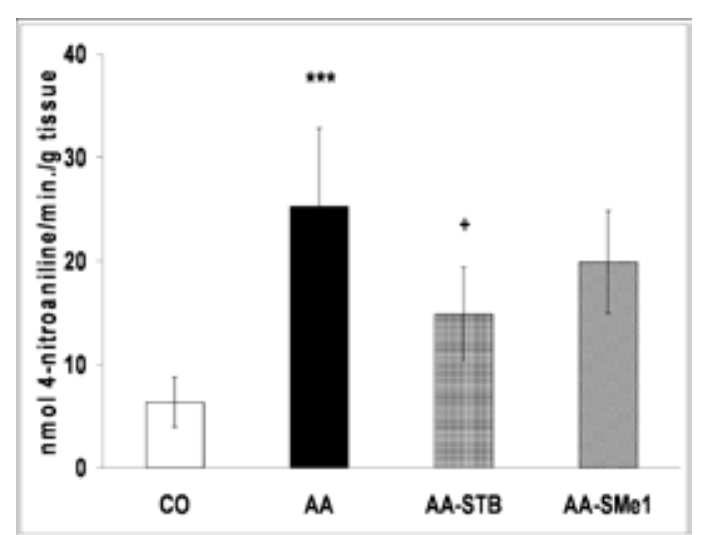

Figure 3. Activity of $\gamma$-glutamyltransferase in joint tissue homogenates of arthritic rats treated with pyridoindoles.

The data were expressed as arithmetic mean with S.E.M. Each group contained 6-8 animals. Statistical significance was evaluated using unpaired Student's $t$-test: ${ }^{* *} P<0.001$ with respect to control healthy animals, $+P<0.05$ with respect to untreated arthritic animals. Groups of animals are the same as in Fig. 1.

Chemiluminescence of whole blood and neutrophil count. The production of ROS in rat whole blood, spontaneous and stimulated with PMA, was measured in $250 \mu \mathrm{l}$ samples consisting of $50 \mu \mathrm{l}$ aliquots: luminol $(250 \mu \mathrm{M})$, 200-times diluted rat blood, HRP $(8 \mathrm{U} / \mathrm{ml})$, PMA $(0.01 \mu \mathrm{M})$ and phosphate buffer. CL was evaluated in a microplate luminometer Immunotech LM-01T (Czech Republic) at $37^{\circ} \mathrm{C}$. Data were based on integral values of CL over $3600 \mathrm{~s}$ $(\mathrm{RLU} \times \mathrm{s}$; RLU, relative light units). Neutrophil count was assessed by Coulter Counter (Coulter Electronics, England). Before counting, whole blood was diluted 2000 times and erythrocytes were destroyed by a lysing reagent (Jancinova et al., 2009).

Statistical analysis of data. The data for all parameters are expressed as arithmetic mean and S.E.M. For significance calculations unpaired Student's $t$-test was used with $* P<0.05$ (significant), $* * P<0.01$ (very significant), *** $P<0.001$ (extremely significant). The arthritis group was compared with healthy control animals (*). The treated arthritis groups were compared with untreated arthritis $(+)$.

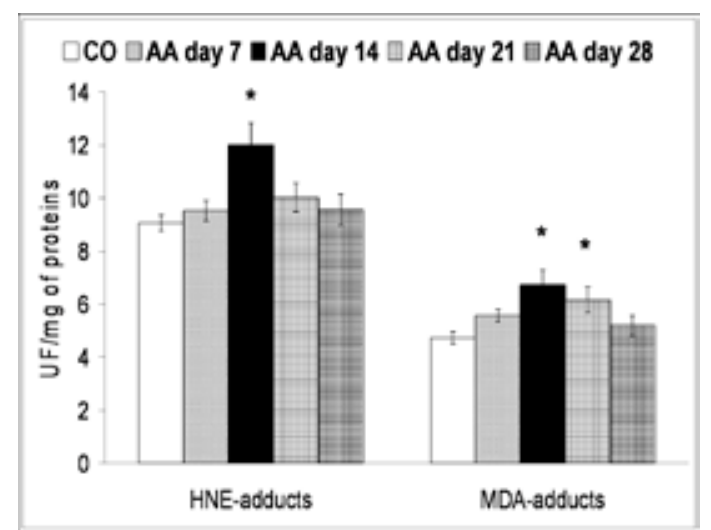

Figure 5. Level of HNE- and MDA-protein adducts in plasma of arthritic rats monitored in time profile (day 7, 14, 21 and 28).

The data were expressed as arithmetic mean with S.E.M. Each group contained 6-8 animals. Statistical significance was evaluated using unpaired Student's $t$-test: ${ }^{*} P<0.05$ with respect to control healthy animals. The experiment included healthy intact animals as reference controls (CO), arthritic animals without any drug administration (AA).

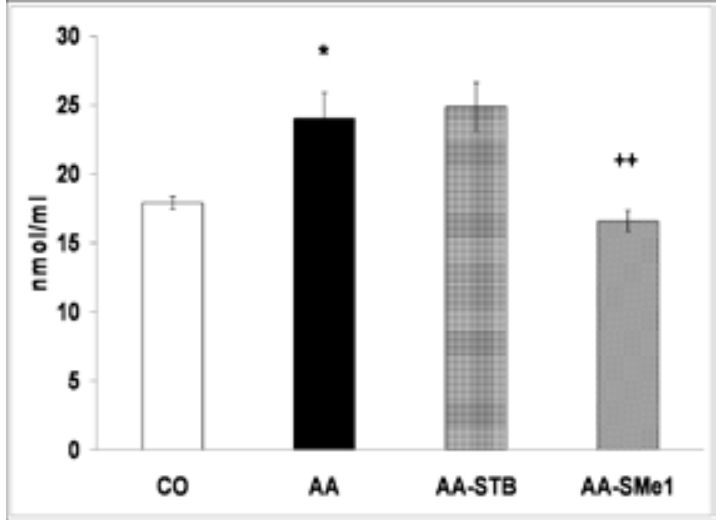

Figure 4. Level of thiobarbituric acid reacting substances in plasma of arthritic rats treated with pyridoindoles.

The data were expressed as arithmetic mean with S.E.M. Each group contained 6-8 animals. Statistical significance was evaluated using unpaired Student's $t$-test: ${ }^{*} P<0.05$ with respect to control healthy animals, $++P<0.01$ with respect to untreated arthritic animals. Groups of animals are the same as in Fig. 1.

\section{RESULTS}

We tested the efficacy of two PI antioxidants in reducing the oxidative stress caused by auto-immune inflammatory processes in the rat adjuvant arthritis model. Both PI studied significantly increased the body mass of animals (Fig. 1) and moderately corrected the HPV (Fig. 2).

STB, but not SMe1 significantly decreased the activity of GGT in joint homogenates (Fig. 3). However, SMe1 was more effective in decreasing plasma levels of TBARS (Fig. 4).

Both HNE- and MDA-protein adducts were noticeably elevated during AA progression on day 14, while MDA-protein adducts were elevated also on day 21. Thus we decided to monitor the HNE- and MDAprotein adducts on day 14 (Fig. 5). STB was more effective than SMe1 in decreasing HNE- and MDAprotein adducts in plasma on day 14, especially the MDA-protein adducts (Fig. 6).

We found that PI reduced the biochemical markers associated with OS and inflammation (TBARS, HNE-

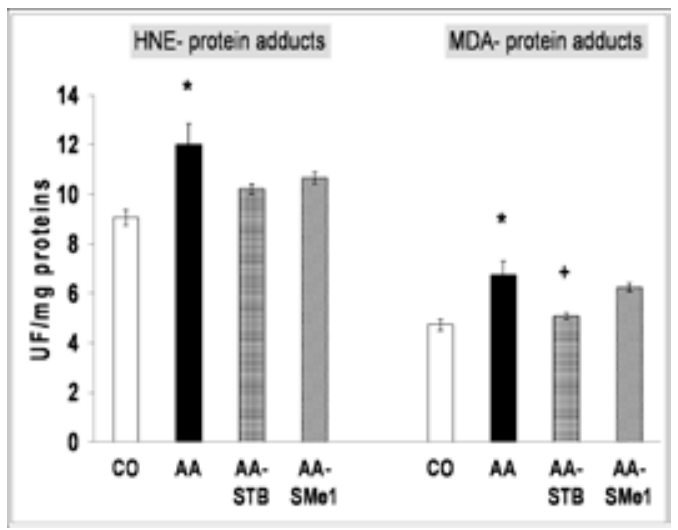

Figure 6. Effect of pyridoindole treatment on the level of plasmatic HNE- and MDA-protein adducts measured on day 14.

The data were expressed as arithmetic mean with S.E.M. Each group contained 6-8 animals. Statistical significance was evaluated using unpaired Student's $t$-test: ${ }^{*} P<0.05$ with respect to control healthy animals, $+P<0.05$ with respect to untreated arthritic animals. Groups of animals are the same as in Fig. 1. 


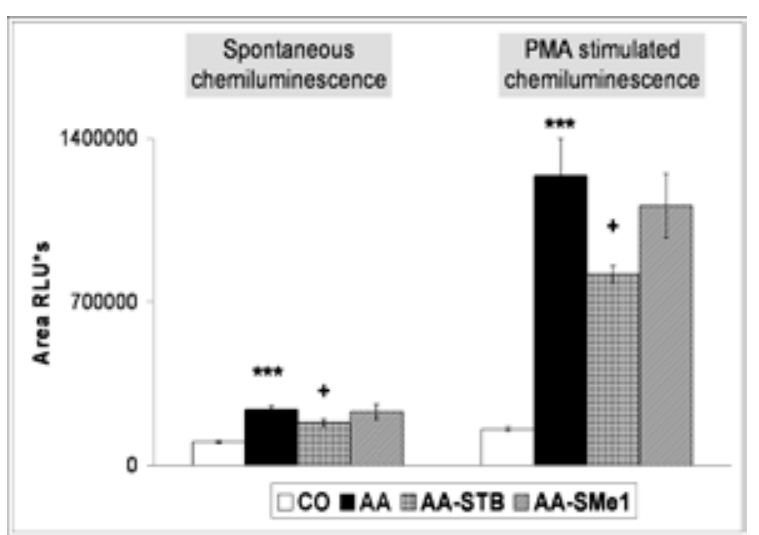

Figure 7. Spontaneous and PMA stimulated whole blood chemiluminescence in rats with AA. Effect of pyridoindole treatment. The data were expressed as arithmetic mean with S.E.M. Each group contained 6-8 animals. Statistical significance was evaluated using unpaired Student's $t$-test: ${ }^{* * *} P<0.001$ with respect to control healthy animals, $+P<0.05$ with respect to untreated arthritic animals. Groups of animals are the same as in Fig. 1.

and MDA-protein adducts, and GGT). STB, but not SMe1, significantly decreased spontaneous and PMAstimulated chemiluminescence, and also reduced neutrophil count in whole blood (Fig. 7 and 8). STB was more effective than SMe1 in reducing the markers of OS.

\section{DISCUSSION}

Numerous studies have suggested a role of oxidative stress in the pathogenesis of RA (Bauerova \& Bezek, 1999; Bohanec et al., 2009). Several clinical studies as well as preclinical animal models of RA have documented an imbalance in the body's reduction/oxidation homeostasis to a more pro-oxidative environment, suggesting that therapies which restore the redox balance may have beneficial effects on the disease process. The role of ROS in the etiology of RA is supported by numerous studies documenting that not only the damaging effects of ROS but also the role that ROS play in regulating various inflammatory processes contributes to the pathogenesis of the disease (Kunsch et al., 2005). The net effect of redox signaling are highly specific changes in gene expression and in the cellular phenotype. Therefore, by serving as second messengers, ROS/RNS are not only toxic agents but also mediators of physiological function (Giustarini et al., 2004; Poli et al., 2004). GGT, as a non-specific marker of inflammation and oxidative stress, has been assessed in different cells and tissues of the lymphatic system - T-lymphocytes, macrophages, spleen and thymus tissue (Koner et al., 1997). The ectoenzyme form of GGT is not present on non-active peripheral T-lymphocytes, but its expression rises after activation of native T-lymphocytes. In other tissues, GGT is essential for „scavenging” glutathione metabolites (mostly $\gamma$-glutamyl) and their transport into cells, where GSH is synthesized de novo (Carlisle et al., 2003). GGT is an important component of inflammatory processes since its activity is closely connected with the overall antioxidant status of the organism. We found that the activity of GGT was approx. 3-6 times higher in AA animals than in healthy controls in the spleen and 1.4-2.3 higher in the joint (Bauerova et al., 2006; 2008b; 2009; Sotnikova et al., 2009). We assume that the increased activity of

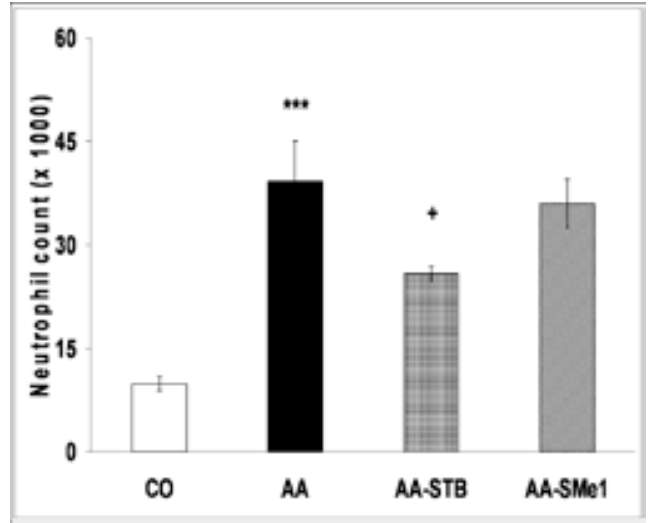

Figure 8. Neutrophil count $1 \mu \mathrm{l}$ of whole blood in arthritic rats treated with PI.

The data were expressed as arithmetic mean with S.E.M. Each group contained 6-8 animals. Statistical significance was evaluated using unpaired Student's $t$-test: ${ }^{* *} P<0.001$ with respect to control healthy animals, $+P<0.05$ with respect to untreated arthritic animals. Groups of animals are the same as in Fig. 1.

GGT in AA is a result of elevated systemic oxidative stress. In the present experiment the GGT activity is also elevated in peripheral joint tissue (Fig. 3). This finding is in good agreement with clinical studies of patients with RA who had increased levels of GGT not only in the serum and urine but also in synovial fluid (Rambabu et al., 1990). In one of our studies we showed a good correlation between the GGT activity in joint tissue and the hind paw volume of arthritic animals (Bauerova et al., 2006). STB was significantly effective in suppressing the increased activity of GGT in the joint, as expected from its antioxidant potential. Moreover, this finding could be considered as an indirect proof of its good distribution into peripheral tissues and supports previous results on STB pharmacokinetics in rats (Kallay et al., 1990; 1991). A frequently used marker of lipid peroxidation is MDA assessed as an adduct with TBA. Clinical studies have shown increased plasmatic levels of MDA in patients with RA (Baskol et al., 2005; 2006; Sarban et al., 2005). In animal models of AA, the level of MDA in the plasma of arthritic animals was elevated (Tastekin et al., 2007; He et al., 2006; Bauerova et al., 2008b; 2009; Strosova et al., 2008; 2009; Sotnikova et al., 2009). Interestingly, SMe1 was more effective in decreasing the plasmatic level of TBARS than was STB; to understand this difference better further investigation is required. As SMe1 did not influence the GGT activity in joint homogenates, but reduced the plasmatic level of TBARS, we assumed that it affected primarily the lipid peroxidation. Oxidation of proteins was influenced only mildly as could be seen from the SMe1 effect on the level of HNE- and MDA-protein adducts in the plasma. In recent years, methods based on chemiluminescence and fluorescence measurements have been widely used for determination of ROS, RNS and lipid peroxidation metabolites. Fluorescent protein adducts are derivatives formed by reaction of secondary metabolites of lipid peroxidation (especially HNE and MDA) with free amino groups of proteins (Requena et al., 1996; Aldini et al., 2007). In murine and human serum, albumin protein fraction was identified as the most fluorescent fraction of proteins (Tsuchida et al., 1985). Several authors have suggested that protein adducts with secondary metabolites of lipid peroxidation also have immunogenic properties and may play a significant role in the pathogenesis 
of auto-immune diseases including RA (Tuma, 2002; Kurien et al., 2006). This highlights the importance of monitoring these adducts in auto-immune, chronic inflammatory diseases. In rat $\mathrm{AA}$, we were the first to monitor the HNE- and MDA-protein adducts in a time profile. The level of HNE-adducts was slightly increased already on day 7. The maximal level of HNE-adducts was reached on day 14, and then it slowly decreased to the control level (day 28). STB reduced the plasmatic level of HNE-protein adducts only moderately, but the level of MDA-protein adducts was reduced significantly (Fig. 6). This effect of STB supports previous finding about its anti-lipid peroxidation properties in vitro (Horakova et al., 2000; Gallova \& Szalayova, 2004) and in vivo (Ulusu et al., 2003). The changes in the level of MDA adducts during the experiment were very similar, except that it was still significantly elevated on day 21 . The time course of lipid peroxidation measured by the MDA-protein adducts resembles the temporal pattern production of ROS by PMA-stimulated neutrophils measured by chemiluminescence of whole blood of arthritic animals, with maximum on day 14 and 21 (Nosal et al., 2007). Recent evidence from animal models of RA emphasizes the importance of neutrophils in the initiation and progression of AA. ROS and RNS are of great relevance in inflammatory and immune processes involved in the pathogenesis of RA (Cross et al., 2006). There are multiple experimental studies dedicated to neutrophil-generated chemiluminescence of whole blood (Arnhold et al., 1994; Miesel et al., 1996; Cedergren et al., 2007) and of synovial fluid (Arnhold et al., 1994; Cedergren et al., 2007), depending on the disease severity in patients with RA. An increase in whole blood chemiluminescence (2-8 times) was shown in RA patients compared with healthy volunteers (Miesel et al., 1996). The results published by Nosal et al. (2007) are in good agreement with this finding. Control AA animals had significantly elevated spontaneous chemiluminescence from the seventh experimental day until the end of experiment (day 28). Neutrophils in whole blood of AA animals reacted excessively to stimulation with PMA and produced 6-9 fold more ROS. The development of AA in rats was also accompanied with an increase in blood neutrophil count when compared with control animals (Nosal et al., 2007). STB proved effective in decreasing the spontaneous and PMA-stimulated chemiluminescence of whole blood of arthritic animals (Fig. 7). This may be explained by its ability to reduce arthritis-caused neutrophil count elevation (Fig. 8). However, other mechanisms can also be involved. STB was found to be a potent inhibitor/scavenger of extracellularly produced ROS in human neutrophils. Interference of STB with ROS as well as with signaling events resulting in NADPH-oxidase activation and myeloperoxidase liberation has been reported (Drabikova et al., 2007). STB and its derivatives were tested in several models of organ ischemia/reperfusion injury where also parameters of OS were monitored. The protective effect of STB was confirmed in various tissues (Knezl et al., 1999; Bezek \& Juranek, 2000; Guz et al., 2007). These promising results with STB at the level of organ injury together with our results in AA present STB as an effective antioxidant. PI in rat AA improved the CBM of animals, however, HPV was affected only moderate. In adjuvant arthritis the level of proinflammatory cytokines IL-1 and TNF $\alpha$ is significantly elevated. These cytokines are also involved in regulation of fever and cachexic processes (Dayer et al., 2002). Mass loss of animals is primarily connected with immunological processes. Concerning the reduction of OS during AA, STB was more effective than SMe1 (activity of GGT, MDAproteins adducts, spontaneous and PMA- stimulated chemiluminescence and neutrofil count in whole blood). The situation for adjuvant arthritis is complicated due to the dominant involvement of Th-1-driven auto-immune ethiopatology. OS in this animal model occurs as a reaction on auto-immune processes. Under these conditions, control of OS is of secondary importance, although it could enhance immuno-modulatory therapy of RA.

\section{Acknowledgements}

The authors thank Mrs. Denisa Komendova for excellent technical assistance.

This work was supported by grants APVV-51-017905, APVV-21-055205, VEGA 02/0090/08, COST B35.

\section{REFERENCES}

Aldini G, Dalle-Donne I, Facino RM, Milzani A, Carini M (2007) Intervention strategies to inhibit protein carbonylation by lipoxidationderived reactive carbonyls. Med Res Rev 27: 817-868.

Arnhold J, Sonntag K, Sauer H, Häntzschel H, Arnold K (1994) Increased native chemiluminescence in granulocytes isolated from synovial fluid and peripheral blood of patients with rheumatoid arthritis. J Biolumin Chemilumin 9: 79-86.

Baskol G, Demir H, Baskol M, Kilic E, Ates F, Kocer D, Muhtaroglu S (2005) Assessment of paraoxonase 1 activity and malondialdehyde levels in patients with rheumatoid arthritis. Clin Biochem 38: 951-965.

Baskol G, Demir H, Baskol M, Kilic E, Ates F, Karakukcu C, Ustdal M (2006) Investigation of protein oxidation and lipid peroxidation in patients with rheumatoid arthritis. Cell Biochem Funct 24: 307-311.

Bauerova K, Bezek A (1999) Role of reactive oxygen and nitrogen species in etiopathogenesis of rheumatoid arthritis. Gen Physiol Biophys 18: $15-20$.

Bauerova K, Nosalova V, Mihalova D, Navarova J (2004) Contribution to safe anti-inflammatory therapy with indomethacin. Cent Eur J Public Health 12: S8-S10.

Bauerova K, Kucharska J, Mihalova D, Navarova J, Gvozdjakova A, Sumbalova Z (2005a) Effect of coenzyme $Q_{10}$ supplementation in the rat model of adjuvant arthritis. Biomed Pap 149: 501-503.

Bauerova K, Valentova J, Ponist S, Navarova J, Komendova D, Mihalova D (2005b) Effect of copper complexes on the development of adjuvant arthritis: therapeutic and toxicological aspects. Biologia 60: $67-70$.

Bauerova K, Ponist S, Ondrejickova O, Komendova D, Mihalova D (2006) Association between tissue gamma glutamyl-transferase and clinical markers of adjuvant arthritis in Lewis rats. Neuro Endocrinol Lett 27: 172-175.

Bauerova K, Kucharska J, Ponist S, Gvozdjakova A (2008a) Coenzyme $\mathrm{Q}_{10}$ supplementation in adjuvant arthritis (pre-clinical study). In $M i$ tochondrial medicine: mitochodrial metabolism, diseases, diagnosis and therapy. Gvozdjakova A, eds, pp 340-342. Springer, Netherlands.

Bauerova K, Ponist S, Navarova J, Dubnickova M, Paulovicova E, Pajtinka M, Kogan G, Mihalova D (2008b) Glucomannan in prevention of oxidative stress and inflammation occuring in adjuvant arthritis. Neuro Endocrinol Lett 29: 101-106.

Bauerova K, Paulovicova E, Mihalova D, Svik K, Ponist S (2009) Study of new ways of supplementary and combinatory therapy of rheumatoid arthritis with immunomodulators. Glucomannan and imunoglukan in adjuvant arthritis. Toxicol Ind Health 25: 329-335.

Bezek S, Juranek I (2000) Effects of stobadine on hypoxia and hypoxia/reoxygenation injury in isolated hepatocytes from fasted rats. Biologia 55: 5-8.

Biasi F, Bosco M, Lanfranco G, Poli G (1995) Cytolysis does not per se induce lipid peroxidation: Evidence in man. Free Radic Biol Med 18: 909-912

Bohanec Grabar P, Logar D, Tomsic M, Rozman B, Dolzan V (2009) Genetic polymorphisms modifying oxidative stress are associated with disease activity in rheumatoid arthritis patients. Dis Markers 26: $41-48$.

Brown RK, Kelly FJ (1996) Peroxides and other products. In: Free Radicals, a practical approach. Punchard NA, Kelly FJ, eds, pp 119-131. Oxford University Press, Oxford.

Carlisle ML, King MR, Karp DR (2003) gamma-Glutamyl transpeptidase activity alters the $T$ cell response to oxidative stress and Fasinduced apoptosis. Int Immunol 15: 17-27.

Cedergren J, Forslund T, Sundqvist T, Skogh (2007) Intracellular oxidative activation in synovial fluid neutrophils from patients with 
rheumatoid arthritis but not from other arthritis patients. I Rheumatol 34: 2162-2170.

Cross A, Barnes T, Bucknall RC, Edwards SW, Moots RJ (2006) Neutrophil apoptosis in rheumatoid arthritis is regulated by local oxygen tensions within joints. J Leukoc Biol 80: 521-528.

Dayer JM (2002) Interleukin 1 or tumor necrosis factor-alpha: which is the real target in rheumatoid arthritis? J Rheumatol (Suppl) 65: 10-15.

Drabikova K, Jancinova V, Nosal R, Pecivova J, Macickova T, Turcani P (2007) Inhibitory effect of stobadine on FMLP-induced chemiluminescence in human whole blood and isolated polymorphonuclear leukocytes. Luminescence 22: 67-71.

Drabikova K, Perecko T, Nosal R, Baureova K, Ponist S, Mihalova D, Kogan G, Jancinova V (2009) Glucomannan reduces neutrophil free radical production in vitro and in rats with adjuvant arthritis. Pharmacol Res 59: 399-403.

Firestein GS (2003) Evolving concepts of rheumatoid arthritis. Nature 423: $356-361$.

Gallova J, Szalayova S (2004) The effect of stobadine on the copperinduced peroxidation of egg yolk phosphatidylcholine in multilamellar liposomes. Gen Physiol Biophys 23: 297-306.

Giustarini D, Rossi R, Milzani A, Colombo R, Dalle-Donne I (2004) S-Glutathionylation: from redox regulation of protein functions to human diseases. J Cell Mol Med 8: 201-212.

Guz G, Demirogullari B, Ulusu NN, Dogu C, Demirtola A, Kavutcu M, Omeroglu S, Stefek M, Karasu C (2007) Stobadine protects rat kidney against ischaemia/reperfusion injury. Clin Exp Pharmacol Physiol 34: 210-216.

Gvozdjakova A, Kucharska J, Tanaka S, Neradova B, Bauerova K (2004) Coenzyme Q10 supplementation differently modulated heart and skeletal muscle mitochondrial function induced by adjuvant arthritis. Mitochondrion 4: 20-21.

He YH, Zhou J, Wang YS, Xiao C, Tong Y, Tang JC, Chan AS, Lu AP (2006) Anti-inflammatory and anti-oxidative effects of cherries on Freund's adjuvant-induced arthritis in rats. Scand J Rheumatol 35: 356-358.

Horakova L, Ondrejickova O, Bachrata K, Vajdova M (2000) Preventive effect of several antioxidants after oxidative stress on rat brain homogenates. Gen Physiol Biophys 19: 195-205.

Jancinova V, Perecko T, Nosal R, Kostalova D, Bauerova K, Drabikova K (2009) Decreased activity of neutrophils in the presence of diferuloylmethane (curcumin) involves protein kinase $C$ inhibition. Eur J Pharmacol 612: 161-166.

Juranek I, Horakova L, Rackova L, Stefek M (2010) Antioxidants in treating pathologies involving oxidative damage: an update on medicinal chemistry and biological activity of stobadine and related pyridoindoles. Curr Med Chem 17: 552-570.

Kallay Z, Bittererova J, Brejcha A, Faberova V, Bezek S, Trnovec T (1990) Plasma concentration, tissue distribution and excretion of the prospective cardioprotective agent cis-(2)-2,3,4,4a,5,9b-hexahydro-2, 8-dimethyl-1H-pyrido-[4,3b] indole dihydrochloride in rats. Arzneimittelforschung 40: 974-979.

Kallay Z, Soltes L, Trnovec T (1991) Pharmacokinetics of stobadine and of the sum of its metabolites in rats during repeated administration. Biopharm Drug Dispos 12: 201-205.

Knezl V, Sotnikova R, Okruhlicova L, Navarova J (1999) Effect of stobadine on cardiac injury induced by ischemia and reperfusion. Life Sci 65: 1931-1933.

Kogan G, Stasko A, Bauerova K, Polovka M, Soltes L, Brezova V, Navarova J, Mihalova D (2005) Antioxidant properties of yeast (1-3)- $\beta$-D-glucan studied by electron paramagnetic resonance spectroscopy and its activity in the adjuvant arthritis. Carbohydr Polym 61: 18-28.

Koner BC, Banerjee BD, Ray A (1997) Effects of stress on gamma glutamyl transpeptidase (GGT) activity in lymphoid system of rats: modulation by drugs. Indian J Exp Biol 35: 222-224.

Kunsch C, Sikorski JA, Sundell CL (2005) Oxidative stress and the use of antioxidants for the treatment of rheumatoid arthritis. Curr Med Chem Immunol Endocr Metab Agents 5: 249-258.
Kurien BT, Hensley K, Bachmann M, Scofield RH (2006) Oxidatively modified autoantigens in autoimmune diseases. Free Radic Biol Med 41: 549-56.

Miesel R, Hartung R, Kroeger H (1996) Priming of NADPH oxidase by tumor necrosis factor alpha in patients with inflammatory and autoimmune rheumatic diseases. Inflammation 20: 427-438.

Nosal R, Jancinova V, Petríkova M, Ponist S, Bauerova K (2007) Suppression of oxidative burst of neutrophils with methotrexate in rat adjuvant arthritis. Chem Listy 101: 243-244.

Ondrejickova O, Ziegelhoeffer A, Gabauer I, Sotnikova R, Styk J, Gibala P, Sedlak J, Horakova L (1993) Evaluation of ischemia-reperfusion injury by malondialdehyde, glutathione and gamma-glutamyl transpeptidase: lack of specific local effects in diverse parts of the dog heart following acute coronary occlusion. Cardioscience 4: 225230.

Orlowski M, Meister A (1970) The $\gamma$-glutamyl cycle: a possible transport system for amino acids. Proc Natl Acad Sci US A 67: 1248-1255.

Poli G, Leonarduzzi G, Biasi F, Chiarpotto E (2004) Oxidative stress and cell signalling. Curr Med Chem 11: 1163-1182.

Requena JR, Fu MX, Ahmed MU, Jenkins AJ, Lyons TJ, Thorpe SR (1996) Lipoxidation products as biomarkers of oxidative damage to proteins during lipid peroxidation reactions. Nephrol Dial Transplant 11: $48-53$.

Rambabu K, Ansari AA, Shaafie IA, Chelvam AP, Ziu MM (1990) Gamma-glutamyl transpeptidase in synovial fluid, serum, and urine of patients with rheumatoid arthritis. Biochem Med Metab Biol 43: 183-192.

Rovensky J, Stancikova M, Svik K, Bauerova K, Jurcovicova J (2009) The effects of beta-glucan isolated from Pleurotus ostreatus on methotrexate treatment in rats with adjuvant arthritis. Rheumatol Int doi: 10.1007/s00296-009-1258-z.

Sarban A, Kocyigit A, Yazar M, Isikan UE (2005) Plasma total antioxidant capacity, lipid peroxidation, and erythrocyte antioxidant enzyme activities in patients with rheumatoid arthritis and osteoarthritis. Clin Biochem 38: 981-986.

Sotnikova R, Ponist S, Navarova J, Mihalova D, Tomekova V, Strosova M, Bauerova K (2009) Effects of sesame oil in the model of adjuvant arthritis. Neuro Endocrinol Lett 30: 22-24.

Stolc S, Bauer V, Benes L, Tichy M (1983) Medicine with antiarrhythmic and antihypoxic activity and its method of preparation. Patents: CS 229 067, SWED. 8204693-9, BELG. 894148, SWISS 651754 , BRD P-3231088, SPAIN 553 017, JAP. 1514040.

Strosova M, Tomaskova I, Ponist S, Bauerova K, Karlovska J, Spickett CM, Horakova L (2008) Oxidative impairment of plasma and skeletal muscle sarcoplasmic reticulum in rats with adjuvant arthritis - effects of pyridoindole antioxidants. Neuro Endocrinol Lett 29: 706-711.

Strosova M, Karlovska J, Spickett CM, Orszaghova Z, Ponist S, Bauerova K, Mihalova D, Horakova L (2009) Modulation of SERCA in the chronic phase of adjuvant arthritis as a possible adaptation mechanism of redox imbalance. Free Radic Res 43: 852-864.

Tastekin N, Aydogdu N, Dokmeci D, Usta U, Birtane M, Erbas H, Ture M (2007) Protective effects of L-carnitine and alpha-lipoic acid in rats with adjuvant arthritis. Pharmacol Res 56: 303-310.

Tsuchida M, Miura T, Mizutani K, Aibara K (1985) Fluorescent substances in mouse and human sera as a parameter of in vivo lipid peroxidation. Biochim Biophys Acta 834: 196-204.

Tuma DJ (2002) Role of malondialdehyde-acetaldehyde adducts in liver injury. Free Radic Biol Med. 32: 303-8.

Ulusu NN, Sahilli M, Avci A, Canbolat O, Ozansoy G, Ari N, Bali M, Stefek M, Stolc S, Gajdosik A, Karasu Ç (2003) Pentose phosphate pathway glutathione-dependent enzymes and antioxidant defense during oxidative stress in diabetic rodent brain and peripheral organs: effect of stobadine and vitamin E. Neurochem Res 28: 815-823. 